\title{
The peculiar horizontal branch morphology of the Galactic globular clusters NGC 6388 and NGC 6441: new insights from UV observations
}

\author{
G. Busso ${ }^{1,2,3}$, S. Cassisi ${ }^{1}$, G. Piotto ${ }^{3}$, M. Castellani ${ }^{4}$, M. Romaniello ${ }^{5}$, M. Catelan ${ }^{6}$, S. G. Djorgovski ${ }^{7}$, \\ A. Recio Blanco ${ }^{8}$, A. Renzini ${ }^{9}$, M. R. Rich ${ }^{10}$, A. V. Sweigart ${ }^{11}$, and M. Zoccali ${ }^{6}$
}

1 INAF - Osservatorio Astronomico di Collurania, via M. Maggini, 64100 Teramo, Italy e-mail: [busso;cassisi]@oa-teramo.inaf.it

2 Institut für Theoretische Physik und Astrophysik, University of Kiel, Leibnizstrasse 15, 24114 Kiel, Germany

3 Dipartimento di Astronomia, Università di Padova, Vicolo dell'Osservatorio 2, 35122 Padova, Italy e-mail: giampaolo.piotto@unipd.it

4 INAF - Osservatorio Astronomico di Roma, via di Frascati 33, 00040, Monte Porzio Catone, Roma, Italy e-mail: m. castellani@mporzio.astro.it

5 European Southern Observatory, Karl Schwarzchild Strasse 2, 85748 Garching, Germany e-mail: mromanie@eso.org

6 Pontificia Universidad Católica de Chile, Departamento de Astronomía y Astrofísica, Av. Vicuña Mackenna 4860, 782-0436 Macul, Santiago, Chile e-mail: [mcatelan;mzoccali]@astro.puc.cl

7 Astronomy Department, California Institute of Technology, MC 105-24, Pasadena, CA 91125, USA e-mail: george@astro.caltech.edu

8 Observatoire de la Côte d'Azur, Dpt. Cassiopée, CNRS/UMR6202, BP 4229, 06304 Nice Cedex 4, France e-mail: arecio@obs-nice.fr

9 INAF - Osservatorio Astronomico di Padova, vicolo Osservatorio 5, 35122 Padova, Italy e-mail: alvio.renzini@oapd.inaf.it

10 Department of Physics and Astronomy, UCLA, 430 Portola Plaza, Los Angeles CA, 90095-1547, USA e-mail: rmr@astro.ucla.edu

11 NASA Goddard Space Flight Center, Code 667, Greenbelt, MD 20771, USA

e-mail: allen.v.sweigart@nasa.gov

Received 7 May 2007 / Accepted 7 August 2007

\section{ABSTRACT}

\begin{abstract}
Context. In this paper we present multiband optical and UV Hubble Space Telescope photometry of the two Galactic globular clusters NGC 6388 and NGC 6441.

Aims. We investigate the properties of their anomalous horizontal branches in different photometric planes in order to shed light on the nature of the physical mechanism(s) responsible for the existence of an extended blue tail and of a slope in the horizontal branch, visible in all the color-magnitude diagrams.

Methods. New photometric data have been collected and carefully reduced. Empirical data have been compared with updated stellar models of low-mass, metal-rich, He-burning structures, transformed to the observational plane with appropriate model atmospheres. Results. We have obtained the first UV color-magnitude diagrams for NGC 6388 and NGC 6441. These diagrams confirm previous results, obtained in optical bands, about the presence of a sizeable stellar population of extremely hot horizontal branch stars. At least in NGC 6388, we find a clear indication that at the hot end of the horizontal branch the distribution of stars forms a hook-like feature, closely resembling those observed in NGC 2808 and Omega Cen. We briefly review the theoretical scenarios that have been suggested for interpreting this observational feature. We also investigate the tilted horizontal branch morphology and provide further evidence that supports early suggestions that this feature cannot be interpreted as an effect of differential reddening. We show that a possible solution of the puzzle is to assume that a small fraction - ranging between 10-20\% - of the stellar population in the two clusters is strongly helium-enriched ( $Y \sim 0.40$ in NGC 6388 and $Y \sim 0.35$ in NGC 6441). The occurrence of a spread in the He abundance between the canonical value $(Y \sim 0.26)$ and the quoted upper limits can significantly help in explaining the "whole" morphology of the horizontal branch and the pulsational properties of the variable stars in the target clusters.
\end{abstract}

Key words. stars: horizontal-branch - ultraviolet: stars - globular clusters: individual: NGC 6388 globular clusters: individual: NGC 6441

\section{Introduction}

The study of galactic globular clusters (GCs) is of paramount relevance in order to understand the process of formation of the Milky Way as well as for its implication for many topics related to stellar evolution theory and, in particular for the investigation of the evolutionary properties of low-mass stars.

Even if we are able in general to provide a detailed interpretation of the various empirical sequences observed in the 
color-magnitude diagrams (CMDs) of Galactic GCs in terms of the evolutionary and structural properties of both $\mathrm{H}$ - and $\mathrm{He}$ burning low-mass stars, many important issues still wait for a clear comprehension. One of the most relevant issues is related to the long-standing problem of the 2 nd parameter concerning the morphology of the horizontal branch (HB) (see Catelan 2007, and references therein). In these past few years, it is becoming more and more evident that we need to revise our point of view concerning the "nature" of 2 nd parameter: there is increasing evidence that different 2 nd parameters could be at work in different GCs, as well as that more than a "single" parameter besides metallicity (the first parameter) - could be at work in the same cluster (e.g., Lee et al. 2005, and references therein). In this context, a strong contribution has been provided by the observational data for the two metal-rich $([\mathrm{Fe} / \mathrm{H}] \approx-0.5)$ bulge clusters NGC 6388 and NGC 6441 collected by Rich et al. (1997) as part of a Hubble Space Telescope (HST) project. The optical photometric data collected by Rich et al. disclosed a number of important and unexpected empirical facts:

- both clusters show not only the red HB, characteristic of metal-rich GCs as 47 Tuc $([\mathrm{Fe} / \mathrm{H}]=-0.76$, Harris 1996), but also a quite extended blue HB. The effective temperature $\left(T_{\text {eff }}\right)$ reached by the blue HB stars in both clusters is so high that their $F 555 \mathrm{~W}$ magnitudes are dominated by the huge bolometric corrections, and the $(F 439 W-F 555 W)$ color index is no more a good $T_{\text {eff }}$ indicator. As a consequence, the blue HB (BHB) appears as a vertical branch, this occurrence hampering a more detailed investigation of the hot $\mathrm{HB}$ stellar populations in these clusters. The fact that NGC 6388 and NGC 6441 show not only the "canonical" stubby red HB, but also an extended blue tail, was the first sound evidence that the second parameter is also at work in old, metal-rich stellar systems;

- even more surprisingly, the CMD of Rich et al. (1997) clearly shows that the mean HB brightness at the top of the blue $\mathrm{HB}$ tail is roughly 0.5 magnitudes brighter in the $F 555 \mathrm{~W}$ band than the red HB portion, which appears significantly sloped as well. This slope, as pointed out in Sweigart \& Catelan (1998) is not present in the theoretical models, and is difficult to explain (see also the discussion in Raimondo et al. 2002).

In an attempt to interpret the occurrence of the blue tail along the $\mathrm{HB}$ as well as of its tilted morphology, many non-canonical scenarios and/or observational effects have been suggested (e.g. Sweigart \& Catelan 1998; Raimondo et al. 2002; Moehler \& Sweigart 2006b; Caloi \& D' Antona 2007; see also Catelan 2007, for a review). We will enter in more details into this topic in the following discussion.

In the last few years, a large effort has also been devoted to the investigation of the properties of the population of variable stars in both clusters (e.g. Pritzl et al. 2002; Clementini et al. 2005; Corwin et al. 2006; Matsunaga et al. 2006). An important and unexpected result - somehow related to the peculiar HB morphology of NGC 6388 and NGC 6441 - was the evidence that their RR Lyrae populations do not behave as in typical Oosterhoff type I systems, as should have been expected on the basis of their metal content. On the contrary, these analyses have shown the presence of a significant fraction of long-period c-type RR Lyrae, which are not commonly seen in most other globular clusters (Catelan 2004, and references therein), while in NGC 6388, but not in NGC 6441, some short-period c-type RR-Lyrae (i.e., with $P \leq 0.3 \mathrm{~d}$ ) have been observed.
Table 1. Fundamental parameters of NGC 6388 and NGC 6441*.

\begin{tabular}{ccc}
\hline \hline & NGC 6388 & NGC 6441 \\
\hline$l$ & 345.56 & -353.53 \\
$b$ & -6.74 & -5.01 \\
$d_{\odot}$ & 10.0 & 11.7 \\
{$[\mathrm{Fe} / \mathrm{H}]$} & -0.60 & -0.53 \\
$E(B-V)$ & 0.37 & 0.47 \\
\hline
\end{tabular}

${ }^{*} l$ and $b$ are respectively galactic longitude and latitude (in degrees), $d_{\odot}$ is the distance from the Sun (in $\mathrm{kpc}$ ), $[\mathrm{Fe} / \mathrm{H}]$ is the metallicity, and $E(B-V)$ is the reddening (from the latest version of the Harris 1996 catalog).

According to Layden et al. (1999) and Pritzl et al. (2002), the morphology of the light curves of the c-type RR Lyrae in both clusters seems also to be peculiar when compared with that of the first-overtone RR Lyrae observed in other clusters, which must be due to peculiar values of some physical parameters (such as chemical composition, envelope mass). In addition to harboring this anomalous population of RR Lyrae, NGC 6388 and NGC 6441 contain a significant number of type II Cepheids, making them the most metal-rich clusters known to contain this kind of variables.

In summary, it is clear that NGC 6388 and NGC 6441 seem to be very unusual clusters in many respects, and their observational characteristics, in particular the photometric properties, and their RR Lyrae populations severely challenge both the evolutionary and pulsational models of low-mass, metal-rich stellar structures.

With the aim of better understanding the observational properties of the hot HB stars belonging to these clusters, in this work we present the results of an HST project (GO8718, PI: G. Piotto) devoted to collect HST WFPC2 multiband photometry in the filters: $F 255 W, F 336 W, F 439 W$ and $F 555 W$. The main parameters of NGC 6388 and NGC 6441, as given by Harris (1996, in the 2003 revision), are summarized in Table 1.

The data presented in this paper represent an extension of the original $F 439 W-F 555 W$ vs. F555W CMDs published by Rich et al. (1997) and Piotto et al. (2002). Because of the strong bolometric correction and temperature insensitivity, the optical data are not as useful in the study of the hottest HB stars, which can be better studied with the UV observations discussed in the next sections.

\section{Observations and data reduction}

Table 2 summarizes the most relevant information on the observational data set used in the present paper. The data come from HST/WFPC2 observations; in all cases, the PC camera was centered on the cluster center. The images were processed following the recipe in Silbermann et al. (1996): the vignetted pixels and bad pixels and columns were masked out using a vignetting frame created by P. B. Stetson, together with the appropriate data-quality file for each frame. The single chip frames were extracted from the 4-chip-stack files and analyzed separately.

The photometric reduction was carried out using the DAOPHOTII/ALLFRAME package (Stetson 1987). The F439W and $F 555 W$ data were reduced within the snapshot project (for more details, see Piotto et al. 2002). As for the F255W and F336W data, for each image we calculated the appropriate Point Spread Function (PSF). The stars used to calculate the PSF were selected carefully, as in the UV images, especially in the $F 255 \mathrm{~W}$ band, where many cosmic rays were present. 
Table 2. Log of the observations.

\begin{tabular}{lcccc}
\hline \hline Cluster & Filter & Number images & $t_{\exp }[\mathrm{s}]$ & Program ID \\
\hline NGC 6388 & $F 255 W$ & 7 & $1 \times 1100,3 \times 1300,3 \times 1400$ & GO8718 \\
& $F 336 W$ & 3 & $1 \times 260,2 \times 400$ & GO8718 \\
& $F 439 W$ & 3 & $1 \times 50,2 \times 160$ & GO6095 \\
& $F 555 W$ & 2 & $1 \times 12,1 \times 50$ & GO6095 \\
\hline NGC 6441 & $F 255 W$ & 7 & $1 \times 1100,3 \times 1300,3 \times 1400$ & GO8718 \\
& $F 336 W$ & 3 & $1 \times 260,2 \times 400$ & GO8718 \\
& $F 439 W$ & 3 & $1 \times 50,2 \times 160$ & GO6095 \\
& $F 555 W$ & 2 & $1 \times 14,1 \times 50$ & GO6095 \\
\hline
\end{tabular}

With ALLSTAR, we fitted on each image the best PSF, obtaining an approximate list of stars for each single frame. This list was used to match the different frames accurately and to find the correct coordinate transformations among the frames.

ALLFRAME needs also an input star list. We ran this program with two star lists: the first one was obtained from the median image coming from all the $F 255 \mathrm{~W}$ images, and the second one was obtained on the median image of the $F 336 \mathrm{~W}$ frames.

For each of the WFPC2 chips, a catalog of mean magnitudes was created for both $F 255 \mathrm{~W}$ and $F 336 \mathrm{~W}$ bands; for the photometry in the $F 439 W$ and $F 555 W$ bands, we used the HST snapshot data (Piotto et al. 2002). The four photometries were finally combined in order to obtain the various CMDs.

\subsection{The photometric calibration}

In order to calibrate our photometry, we followed the procedure outlined by Dolphin (2000), which allows also to correct the observed magnitudes for charge-transfer efficiency effects (CTE). After having measured the zero point differences between the ALLFRAME magnitudes and the reference aperture of $0.5 \mathrm{arcsec}$, we applied the following calibration equation to transform the magnitudes into the HST Flight Photometric System (STMAG):

$W F P C 2=-2.5 \log \left(\mathrm{DN} \mathrm{s}^{-1}\right)+Z_{\mathrm{FG}}+\Delta Z_{\mathrm{FG}}-\mathrm{CTE}$,

where DN is the flux in digital counts, CTE is the CTE correction, $Z_{\mathrm{FG}}$ is the zero point, different for each filter, and $\Delta Z_{\mathrm{FG}}$ is the zero-point modification for chip and gain settings. Since the $F 255 W$ zero point is available only for the WFPC2 Synthetic System (Holtzman et al. 1995), for consistency we have used the synthetic value also for the $F 336 W$ filter.

\section{The optical and UV CMDs and star counts}

\subsection{The color-magnitude diagrams}

In Fig. 1 we show the optical and UV band CMDs for both clusters. Already a quick look at the two optical CMDs clearly shows the most relevant, common properties of these two clusters, i.e., the occurrence of an extended HB, and the tilt of the HB, with the $\mathrm{HB}$ on the blue side of the instability strip brighter than the branch on the red side. However, the HB morphology in the optical CMDs differs in the two clusters in a number of significant aspects:

- The HB on the blue side of the instability strip appears more populated in NGC 6388 than in NGC 6441. This is not due to a sampling effect, since the number of sampled stars is very similar for the two clusters, as discussed below.

- The blue tail of NGC 6388 seems more extended than in NGC 6441. In NGC 6388 the blue tail extends to more than 1 magnitude fainter than the cluster turn-off (down to $F 555 W \approx 21.5$ ), whereas in the case of NGC 6441 it barely reaches the turn-off brightness, at $F 555 W \approx 20.5$. We note that in this analysis we do not consider the two stars at $F 555 W \approx 22$ as cluster members since they are quite separated from the blue HB tail. Very likely, they do not belong to the cluster but instead to the bulge field population (hot HB stars have been found also in other bulge fields, see Busso et al. 2005).

- In the CMD of NGC 6441 there is a gap at $F 555 W \approx 18.5$, about one magnitude below the HB turning down; no such gap is visible in the HB of NGC 6388, which, instead seems to show a gap at $F 555 W \approx 19.8$. The latter is not present in NGC 6441, but this could be due to the smaller number of stars present in that region of its HB combined with photometric errors, to the lower sensibility to the temperature of the $F 255 W-F 336 W$ color index, as well as to the larger differential reddening affecting this cluster (see discussion in Sect. 4.2). No such gaps are seen in the UV CMDs (see below). However, one should also bear in mind that the occurrence of gaps could be simply due to statistical fluctuations (see the discussion in Catelan et al. 1998).

The right panels of Fig. 1 show the UV CMDs for both clusters. In order to make easier the cross-identification of the same sequences in the optical and UV CMDs, we use different symbols and colors for different stellar groups. In the UV photometry, only the hottest stars are sufficiently bright to be properly measured, namely the blue stragglers (BS), the bluer HB (BHB) stars, the Extreme Horizontal Branch (EHB) stars and the postHB stars. The coolest stars of the red HB clump are totally missed. It is also worth noting how well the BS sequence is separated from the blue HB tail in the UV CMD. This fact is more evident for NGC 6388 than for NGC 6441, possibly as a consequence of the larger differential reddening which characterizes NGC 6441 (see discussion in Sect. 4.2) and the seemingly much larger BS population in the former.

Also the UV CMDs for both clusters show some noteworthy features:

- in both CMDs the brightest stars in the $F 255 \mathrm{~W}$ band are not the stars populating the hottest portion of the blue tail in the optical CMD;

- the gaps visible in the optical bands are not clearly visible in the UV CMDs, making it impossible to establish whether these gaps are real features (as clearly seen in NGC 2808, Sosin et al. 1997; Bedin et al. 2000), or simply a statistical fluctuation in the distribution in temperature along the HB (Catelan et al. 1998). The only possible exception is the abrupt fall down in the NGC $6388 \mathrm{HB}$ star counts at $F 255 W \approx 19.4$, which corresponds to the gap at 


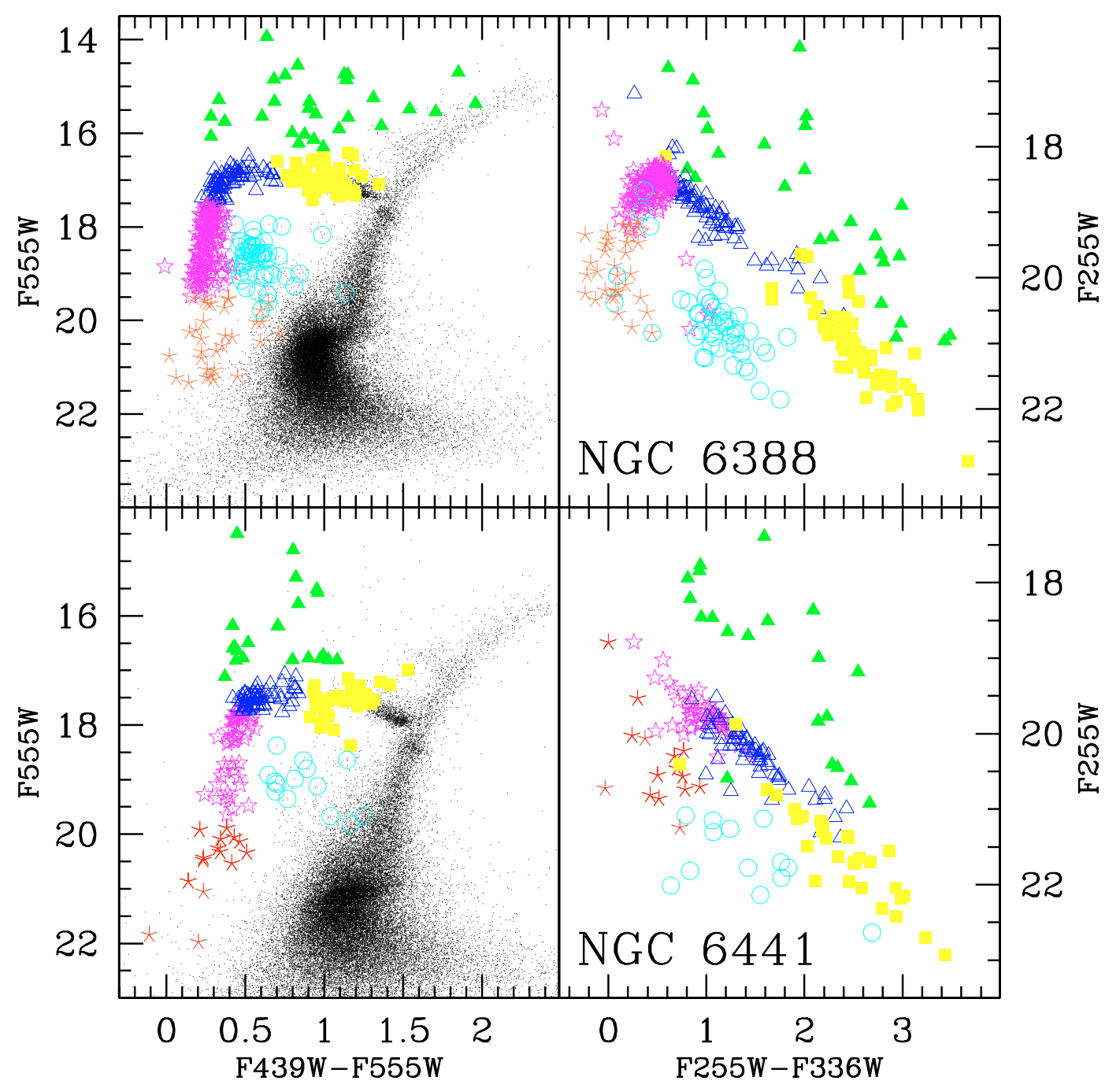

Fig. 1. Optical and UV CMDs for NGC 6388 (upper panels) and NGC 6441 (lower panels). The different symbols and colors refer to the same group of stars appearing both in the optical and UV CMD: yellow-filled squares indicate the red HB, blue open triangles the blue HB, magenta open stars the extended blue HB tail, the filled green triangles the post $\mathrm{HB}$, the cyan open circle the blue stragglers candidates, and the orange asterisks are the blue hook candidates.

$F 555 W \approx 19.8$ in the optical CMD. This feature is relevant for the discussion in Sect. 5;

- there is a large color spread at the hot end of the observed HB sequence, of the order of $\sim 0.5 \mathrm{mag}$ in $F 255 \mathrm{~W}-\mathrm{F} 336 \mathrm{~W}$, larger than the photometric errors (less than $0.1 \mathrm{mag}$ ).

\subsection{Star counts}

Table 3 lists the star counts in a number of relevant parts of the CMD for the two clusters. $N_{\mathrm{RG}}$ is the number of red giant (RG) stars ${ }^{1}$ from the magnitude level corresponding to the average magnitude of the red HB to the tip of the Red Giant Branch (RGB), $N_{\mathrm{HB}}$ is the total number of $\mathrm{HB}$ stars, $N_{\mathrm{rHB}}$ is the number of HB stars redder than the instability strip, $N_{\mathrm{vHB}}$ the number of HB stars within the instability strip (i.e. RR Lyrae stars), $N_{\mathrm{bHB}}$ the number of stars bluer than the instability strip, $N_{\mathrm{gHB}}$ the number of stars bluer than the Grundahl et al. (1999) jump, at $T_{\text {eff }} \sim 11500 \mathrm{~K}$. The HB and RGB have been extracted from

\footnotetext{
${ }^{1}$ It accounts for RGB stars as well as for Asymptotic Giant Branch (AGB) ones. We note that, due to the presence of differential reddening, it would be impossible to distinguish between RGB stars and AGB ones. However, for the present investigation, this is not a relevant problem.
}

the $(F 336 W-F 555 W, F 555 W) \mathrm{CMD}$, as in this diagram it is easier to separate the different CMD parts (see the left panels of Fig. 2), while the star counts $N_{\mathrm{rHB}}, N_{\mathrm{vHB}}$ and $N_{\mathrm{bHB}}$ have been extracted from the $(F 439 W-F 555 W, F 555 W)$ diagram (see the right panels of Fig. 2).

These star counts are surely affected by incompleteness. However, as the two clusters have rather similar HB morphologies, comparable apparent distance moduli, and the observational data have been collected and reduced in the same way, we expect, as a first approximation, similar parts of their CMDs to be affected by similar incompleteness levels. Now, the ratio of the number of RGB stars in NGC 6441 over the number of RGB stars in NGC $6388(1.06 \pm 0.06)$ tells us that we are sampling approximately the same total number of stars in the two clusters. Consistently, also the ratio of the total number of HB stars $(1.00 \pm 0.04)$ is comparable. Interestingly enough, the relative number of red HB stars is the same for the two clusters (ratio $1.06 \pm 0.06$ ).

The relative number of stars within the instability strip is not very informative (too few stars), and it must be noted that this is also the region with the highest contamination by field stars. In any case, we note that NGC 6388 has 22 known RR Lyrae, 
Table 3. Star counts for selected regions of the CMDs of the two clusters (see text for more details).

\begin{tabular}{ccccccc}
\hline \hline & $N_{\text {RGB }}$ & $N_{\mathrm{HB}}$ & $N_{\mathrm{rHB}}$ & $N_{\mathrm{vHB}}$ & $N_{\mathrm{bHB}}$ & $N_{\mathrm{gHB}}$ \\
\hline NGC 6441 & 705 & 1289 & 1122 & 21 & 146 & 61 \\
NGC 6388 & 664 & 1293 & 1056 & 19 & 218 & 154 \\
ratio(NGC 6441/NGC 6388) & $1.06 \pm 0.06$ & $1.00 \pm 0.04$ & $1.06 \pm 0.05$ & $1.11 \pm 0.35$ & $0.70 \pm 0.07$ & $0.40 \pm 0.06$ \\
\hline
\end{tabular}

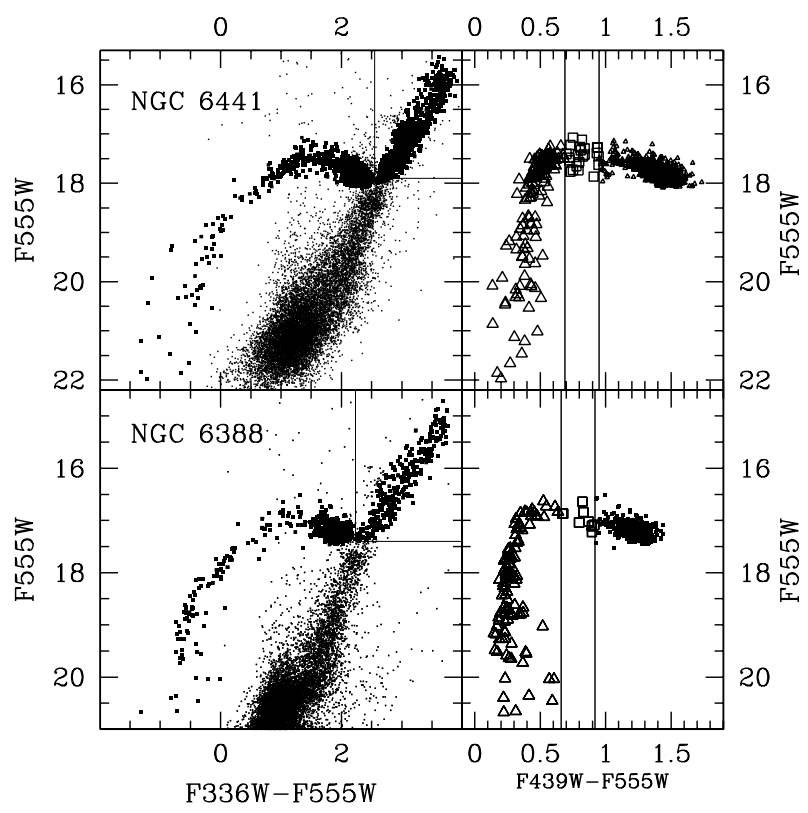

Fig. 2. In the left panels, the $(F 336 W-F 555 W, F 555 W)$ diagrams for both clusters, where the RGB (in the upper right box) and HB selection are shown marked in black. In the right panels, for both clusters the selection in the $(F 439 W-F 555 W, F 555 W)$ for red (points), variable (open squares), and blue (open triangles) HB stars is shown. The two vertical lines represent the RR Lyrae instability strip boundaries $(0.21<$ $F 439 W-F 555 W<0.47)$, from Bono et al. (1997), reddened with the appropriate reddening for each cluster.

while NGC 6441 has 68 RR Lyrae (Corwin et al. 2006), confirming an overabundance of RR Lyrae in the latter. Most interestingly, as suspected from the first visual impression, the blue HB in NGC 6441 is much less populated than in NGC 6388, and the lack of hot stars in NGC 6441 with respect to NGC 6388 is even more pronounced for $T_{\text {eff }} \geq 11500 \mathrm{~K}$, i.e. beyond the Grundahl et al. (1999) jump. Only $11 \pm 1 \%$ of the HB stars of NGC 6441 populate the HB on the blue side of the instability strip, to be compared with the $17 \pm 1 \%$ of blue HB stars in NGC 6388 (the ratio is $0.70 \pm 0.07$ ). NGC 6441 has only $4.7 \pm 0.6 \%$ of its stars hotter than the Grundahl et al. (1999) jump, while $11.9 \pm 1.0 \%$ of the NGC 6388 HB stars are beyond the jump (the ratio is $0.40 \pm 0.06$ ). Whatever the mechanism responsible for the anomalous $\mathrm{HB}$, it apparently acts to a different extent in the two clusters.

\section{The anomalous HBs of NGC 6388 and NGC 6441}

As extensively discussed in the introduction, the HBs of both clusters in the optical CMDs show some relevant features that make them extremely peculiar, i.e. the extension of the HB to very hot temperature and its tilt.

So far, both effects have not been given a firm interpretation (see Catelan 2007, for a recent review). In this work, we try to take advantage of the availability of multi-band photometric data in order to provide additional pieces of information useful for constraining the evolutionary scenario.

In order to perform a detailed comparison between theory and observations, we have adopted the Zero Age Horizontal Branch (ZAHB) models provided by Pietrinferni et al. (2006), supplemented by additional computations performed for this specific project. We have adopted a metallicity $Z=0.008$ and He contents $Y=0.256$. All of the He-burning models used for the present work correspond to an RGB progenitor whose age at the RGB tip is equal to about 13 Gyr. More details about these models ${ }^{2}$ as well as about the adopted physical inputs and numerical assumptions can be found in Pietrinferni et al. (2004, 2006).

Bolometric magnitudes and effective temperatures have been transformed into HST magnitudes and colors according to transformations provided by Origlia \& Leitherer (2000) based on the atmosphere models computed by Bessel et al. (1998).

\subsection{Effects of the reddening in UV bands}

When comparing photometric data with theoretical models, it is necessary to account for reddening and extinction effects. In this context, it is worth noting that both NGC 6388 and NGC 6441 are affected by substantial interstellar extinction (see data in Table 1). As a consequence, an appropriate correction for reddening has to be applied before comparing models with observations.

It is a common procedure to adopt an average extinction law for all stars, regardless of their spectral type. However, the reddening correction depends on the stellar effective temperature. While the commonly adopted approach is surely suitable for stellar systems with a low extinction $[E(B-V) \leq 0.10-0.15]$, it is inappropriate for heavily reddened objects, and the problems increase for blue/UV photometric bands (Grebel \& Roberts 1995).

It is evident that the presence of a large amount of interstellar extinction has no relation at all with the presence of an extended blue tail along the HB of both clusters. However, it could at least partially help explain the other striking feature observed in the HBs, i.e. their tilted morphology.

In order to adopt an accurate reddening correction, we have determined its dependence on $T_{\text {eff }}$ by convolving the appropriate model atmosphere of Bessel et al. (1998) with the filter bandpass, and applying the extinction law of Savage \& Mathis (1979). Figure 3 shows the size of this effect. For the filters $F 555 W$ and $F 439 W$, the extinction correction marginally depends on the stellar $T_{\text {eff }}$, and the average value provided by the table in Holtzman et al. (1995) can be safely used to correct for extinction in these bands. For the bluer filters the difference is larger, and it becomes rather significant for the $F 255 \mathrm{~W}$ band, being of the order of $0.5 \mathrm{mag}$ for $T_{\text {eff }}<10000 \mathrm{~K}$.

The negligible dependence of the extinction in $F 439 \mathrm{~W}$ and $F 555 W$ on effective temperature clearly shows that the sizeable

2 The complete set of evolutionary models can be downloaded from the following URL site:

http://www.oa-teramo.inaf.it/BASTI/index.php 


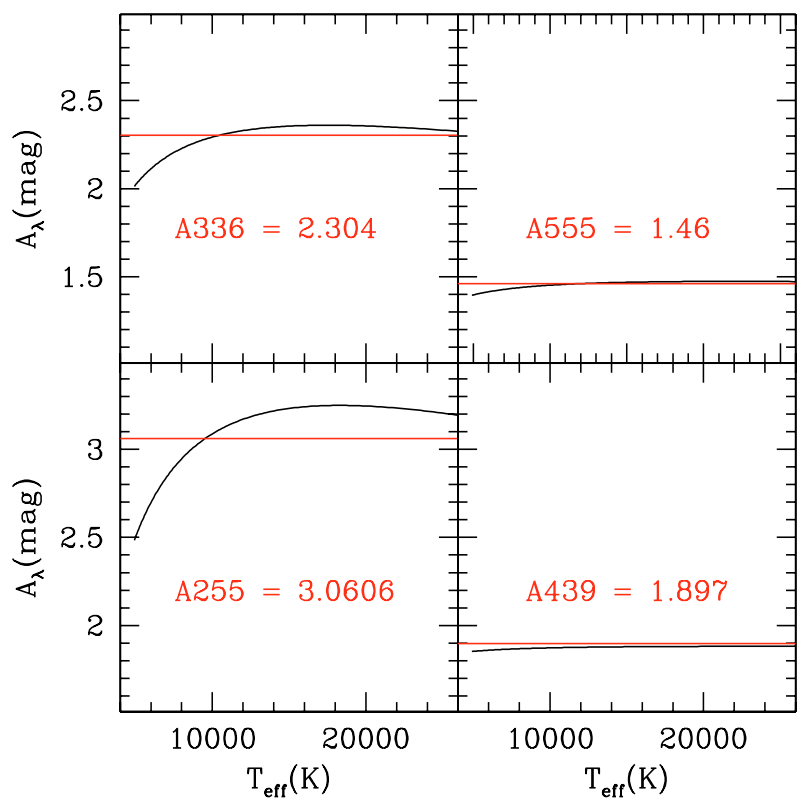

Fig. 3. The extinction coefficients are plotted as a function of the effective temperature for the various photometric bands used in the present work, assuming $E(B-V)=0.45$. The horizontal line shows the average extinction coefficients for the same bands as provided by Holtzman et al. (1995).

interstellar reddening affecting both clusters cannot explain the tilted HB morphology through the effect of $T_{\text {eff }}$ dependence of reddening corrections for the optical bands.

\subsection{Differential reddening}

In the previous section we have shown that a reddening correction depending on the stellar effective temperature is not enough to remove the tilt of the HB. However, our approach assumes an average reddening, while it is well known that both clusters are affected by a sizeable amount of differential reddening (Piotto et al. 1997; Layden et al. 1999; Heitch \& Richtler 1999; Raimondo et al. 2002). We have applied the same procedure used by Raimondo et al. (2002) to both clusters in order to estimate the size of this effect. We divided the Planetary Camera field into 16 regions of $9 \times 9 \operatorname{arcsec}^{2}$, and, for each region, the corresponding CMD has been plotted. We selected one of these CMDs as the reference CMD, and extracted its fiducial points by hand. In Fig. 4 the CMDs for the various regions in the two clusters are compared with the fiducial line obtained by fitting a spline to the fiducial points of the reference CMD. The average displacement in color of the CMD from the fiducial sequence is also listed.

The bottom panel of Fig. 4 is similar to the corresponding figure in Raimondo et al. (2002) for NGC 6441, confirming the presence of differential reddening of the order of 0.10-0.12 mag also on scales of the order of 10 arcsec. The top panel of Fig. 4 shows that also NGC 6388 is affected by differential reddening, though the effect is half as large as in NGC 6441. On the other hand, the slope of the tilted HB in the two clusters is quite similar $[\mathrm{d} V / \mathrm{d}(B-V) \approx 1.5]$, suggesting that differential reddening plays a marginal role in determining the size of the tilt. Such evidence is further supported by the fact that the HB of each box of Fig. 4 shows a slope consistent with that observed for the whole sample $^{3}$, also in those regions where the narrowness of the RGB

3 In a few cases this is not clearly visible, because of the small number of stars in the selected region. suggests that no strong residual differential reddening is present. In summary, as in Sweigart \& Catelan (1998) and Raimondo et al. (2002), we are forced to conclude that the sloped HB is the consequence of an intrinsic property of the $\mathrm{HB}$ stars in NGC 6388 and NGC 6441.

\subsection{The HB morphology in various photometric bands}

In the following discussion, we compare theoretical models with the various CMDs. We adopt the reddening and distance modulus values for which ZAHB models with the canonical $Y=0.256$ best fit the empirical distribution of the red HB clump in the $(F 336 W-F 439 W, F 439 W)$ CMD (see Fig. 5), i.e. $E(F 439 W-$ $F 555 W)=0.45$ and $(m-M)_{F 555 W}=16.65$ for NGC 6388, while for NGC 6441 we adopt $E(F 439 W-F 555 W)=0.48$ and $(m-M)_{F 555 W}=17.4$.

This choice of fitting stellar models to the red clump is clearly arbitrary. In fact, one could in principle try to fit the theoretical predictions to the observations at any point along the HB. This notwithstanding, we consider that the adopted approach for the fitting is reliable for the following reasons: i) in the literature (see the quoted references) devoted to discuss the peculiar features (tilts, jumps, etc.) disclosed by the most recent observations, it is a common procedure to match theoretical ZAHB models to the red HB clump - therefore, for the sake of consistency with previous works we decided to use the same approach; ii) cool ZAHB models should be - at least in principle - more reliable than ZAHB models with an hotter ZAHB location $\left(T_{\text {eff }}\right.$ larger than about $\left.10000 \mathrm{~K}\right)$, since they are not affected by diffusive processes such as atomic diffusion and radiative levitation ${ }^{4}$.

Figure 6 shows a comparison, in the various photometric planes, between the ZAHB models and the HB sequences of NGC 6388 and NGC 6441. For the following discussion we refer only to the HB models with the canonical helium abundance $Y=0.256$, plotted in the CMD with a red line. The comparison between theory and observations clearly shows that it is not possible to have an overall fit of the entire HB: if we impose to the models that they fit the red $\mathrm{HB}$, there is no way they can fit the blue part, and vice versa. The striking evidence is that this is true for all combinations of colors and magnitudes, which raises the suspicion that there must be a real mismatch between the canonical ZAHB models and the observed HBs for both clusters. We wish to note that, so far, this is the first clear empirical evidence showing that the tilted morphology of the HB of the two target GCs is present also in the UV photometric bands - which are much better suited than optical bands for studying hot HB stars.

In passing, we note also that it is not possible to find a unique combination of distance modulus and reddening that allows to properly fit the HB in the various observational planes, even if we consider only the red part of the HB. This occurrence is quite evident in the ( $F 439 W-F 555 W, F 255 W)$ CMD, and it is likely due to the combination of differential reddening and of the fact that for the red portion of the HB we start to lose stars (because they are too faint) when using the $F 255 \mathrm{~W}$ magnitudes.

As already mentioned, when analyzing the optical plane $(F 439 W-F 555 W, F 555 W)$, a tilt is evident when moving from the red HB clump toward bluer colors (see also Rich et al. 1997; Sweigart \& Catelan 1998; and Raimondo et al. 2002), with stars

\footnotetext{
4 On the other hand, one has to keep in mind that cool HB models - once transferred on the various observational planes - could be affected by larger uncertainties eventually present in the adopted color effective temperature relations.
} 

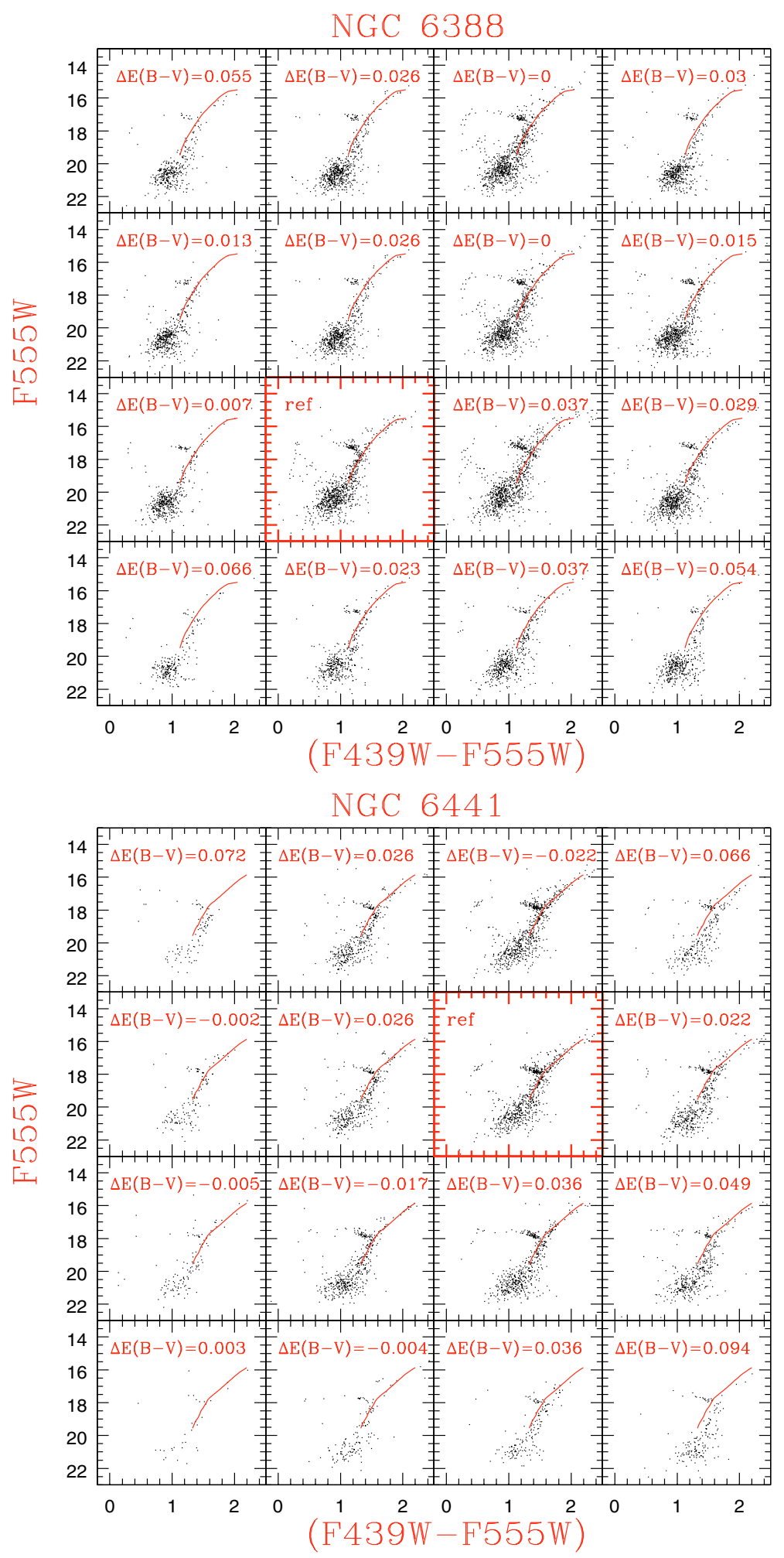

Fig. 4. Top panel: the CMDs of NGC 6388 from the Planetary Camera field divided into 16 cells of $9 \times 9 \operatorname{arcsec}^{2}$. These CMDs have been used to estimate the spatial variation of the reddening in the central region of the cluster. The CMD of the bold panel, labeled with ref, has been arbitrarily adopted as the reference CMD. The reddening relative to the reference CMD is displayed at the top left of each region. Bottom panel: same as top panel but for NGC 6441.

close to the HB turnover which are $\approx 0.5$ mag brighter than the average magnitude of the red HB. It is worth noting once again that this tilt is present in all photometric planes, and it appears even more evident in the UV CMDs, where the blue part of the $\mathrm{HB}$ is up to $\approx 1$ mag brighter than the red part.

It appears - at least in some CMDs, such as the (F336W$F 439 W, F 439 W)$ one in Fig. 5 - that the red portion of the theoretical ZAHB is not able to approach the reddest colors of the observed red HB. Again, this occurrence could be due either to some problems in the adopted color- $T_{\text {eff }}$ relation, or to an effect of the differential reddening affecting these clusters (see the discussion about the change of the red clumpy HB morphology as a consequence of differential reddening made by Raimondo et al. 2002), or to a combination of both effects.

\section{The blue-hook star candidates}

The data shown in Fig. 6 reveal that the models with canonical $Y=0.256$ are not able to reproduce the peculiar morphology of the HB in both clusters. Although this is the most evident 


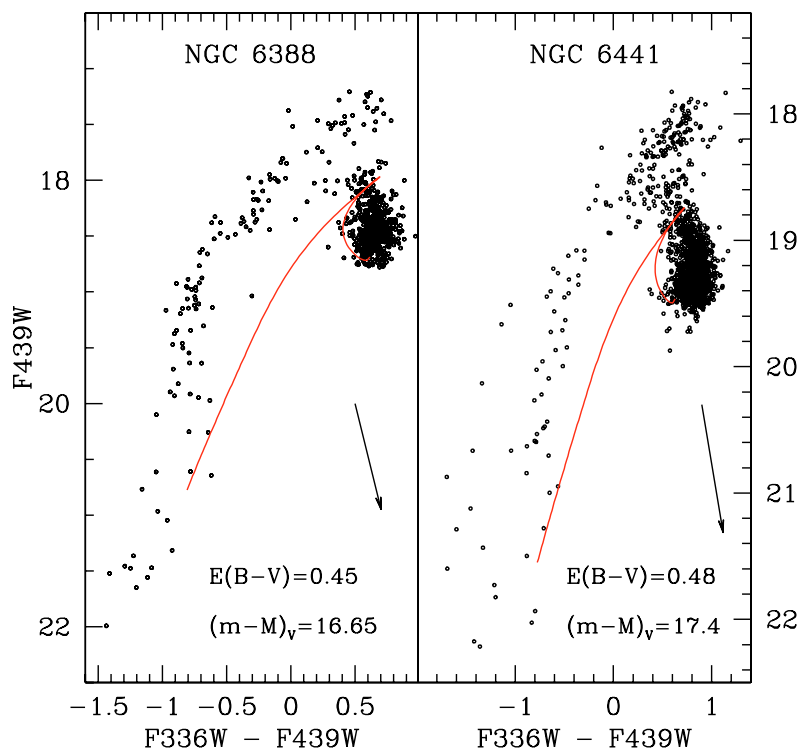

Fig. 5. $(F 336 W-F 439 W, F 439 W)$ CMD of NGC 6388 and NGC 6441. The reddening and distance modulus values determined by best fitting in this diagram the red clump with the canonical ZAHB models for $Y=$ 0.256 are labeled. These canonical ZAHB models are plotted by using a solid line. The arrow represents the reddening vector in this photometric plane. The peculiar shape of the ZAHB sequence, the "glitch" appearing at color $(F 336 W-F 439 W) \sim 0.5$, is due to the fact that this color index is not a monotonic function of the stellar effective temperature, coolward of the Balmer maximum at $T_{\text {eff }} \approx 8500 \mathrm{~K}$.

shortcoming of the models, a careful examination of the same figures shows also that the models are not able to reproduce the color of the hottest HB stars in the CMD. This empirical finding could be interpreted as evidence that the effective temperature of these stars is significantly higher than that of the hottest $\mathrm{HB}$ models (whose total mass is virtually coincident with the He core mass) that can be envisaged on the basis of canonical stellar evolution theory.

This occurrence is evident for NGC 6388, but much less clear in NGC 6441, which is not surprising, since we already noted that the latter has a much less populated and less extended blue HB. The extension of the observed HB beyond the theoretical one is visible in almost all the CMDs of NGC 6388 (Fig. 6), in particular in all the UV CMDs, which are more appropriate for the study of the hottest stars. Again, this mismatch could be an artificial effect of the transformation from the theoretical to the observational plane and/or be due to differential reddening. However, the presence of a possible gap (see Sect. 3), just beyond the location of the hottest stars in the canonical models suggests that the stars observed beyond the gap could have a different nature. Therefore it is tempting to associate them to the blue hook stars already observed by D'Cruz et al. (2000) in Omega Cen (NGC 5139 using far-UV data, and by Brown et al. (2001) in NGC 2808, by means the far-UV and near-UV cameras of the Hubble Space Telescope Imaging Spectrograph.

Although Brown et al. (2001) and Cassisi et al. (2003) have shown that the observational properties of these objects cannot be understood in the framework of canonical stellar evolution theory, we rely on canonical ZAHB models in order to obtain a rough estimate of their effective temperature: they appear to have a $T_{\text {eff }}$ larger than about $25000 \mathrm{~K}$. It is worth noticing that such an effective temperature limit appears significantly lower than the estimated minimum effective temperature (see Brown et al. 2001; and D'Cruz et al. 2000) for blue hook stars. The present analysis does not allow us to verify if this difference is due to the quite approximate approach used for estimating the temperature of these stars, or whether it reflects a real intrinsic difference between blue hook stars in metal-intermediate clusters such as NGC 2808 or Omega Cen and metal-rich ones as our target clusters. The left panels of Fig. 1 of the present paper provide visible evidence supporting the first detection of this feature in metal-rich stellar systems. We tentatively identify as blue-hook candidates all those stars we plot as asterisks.

The blue-hook extension is different in the two clusters: in NGC 6388 the sample of blue hook star candidates is much larger, and its location in the CMD is quite better defined than in the case of NGC 6441. In NGC 6388, the stars belonging to the blue hook show on average increasing color when moving towards fainter magnitudes, while in NGC 6441 the hottest part of the CMD is more dispersed and, on average, the hottest stars have a similar color as the brighter and cooler stars belonging to the same sequence. This occurrence could be due, in part at least, to the larger absolute and differential reddening affecting this cluster. Another possibility to explain the significant color spread in the candidate blue hook stars could be related to the physical mechanism which has been suggested to be at the origin of these peculiar objects, i.e. the so-called delayed He Flash scenario discussed in the following.

As noticed by many authors since Rich et al. (1997), the existence of EHB stars in NGC 6388 and NGC 6441 is quite difficult to be accounted for in the framework of canonical stellar evolution theory, which predicts that the He flash (HeF) takes place at the tip of the Red Giant Branch (RGB). None of these stars would have a hot enough location on the HB able to explain the presence of extended blue tails in any Galactic GCs, though, as recently shown by Castellani et al. (2006), this problem could be less severe than previously claimed. Castellani \& Castellani (1993) suggested the so-called delayed HeF scenario in order to explain the existence of blue tails. This scenario envisages that, as a consequence of a high mass-loss efficiency during the RGB evolution, due to enhanced stellar winds and/or dynamical interactions within dense cluster cores, a stellar structure can lose such a large amount of envelope mass that it fails to ignite the $\mathrm{HeF}$ at the RGB tip, being forced to evolve toward the White Dwarfs (WDs) cooling sequence. Depending on the amount of residual envelope mass, it will still be able to ignite He-burning either at the bright end of the WD cooling sequence (the socalled "early" hot flasher, EHF) or along the WD sequence (the so-called "late" hot flasher, LHF). After the HeF, the star will settle on its ZAHB location, but due to the low mass of the remaining H-rich envelope, its ZAHB location will be significantly hotter than that of canonical stellar models. The first attempt to find a connection between the scenario outlined by Castellani \& Castellani (1993) for EHF stars (see also D'Cruz et al. 1996) and the presence of the blue hook was made by D'Cruz et al. (2000) in Omega Cen, though they found that the predicted ZAHB location of EHFs is not hot enough to explain the empirical evidence.

This scenario has been analyzed also by Sweigart (1997) and more recently by Brown et al. (2001), who devoted particular attention to the evolution of LHFs: this preliminary analysis showed that the convection zone arising with the late $\mathrm{HeF}$, which occurs under conditions of extreme electron degeneracy, is able to penetrate into the $\mathrm{H}$-rich envelope, causing the mixing of $\mathrm{H}$ into the hot He-burning regions (HeF-induced mixing) where it is burnt quickly. A successive dredge-up process of matter that has been processed via both $\mathrm{H}$ - and He-burning would enrich the stellar envelope in helium, carbon and nitrogen. According to the quoted authors, these surface abundance changes would cause 

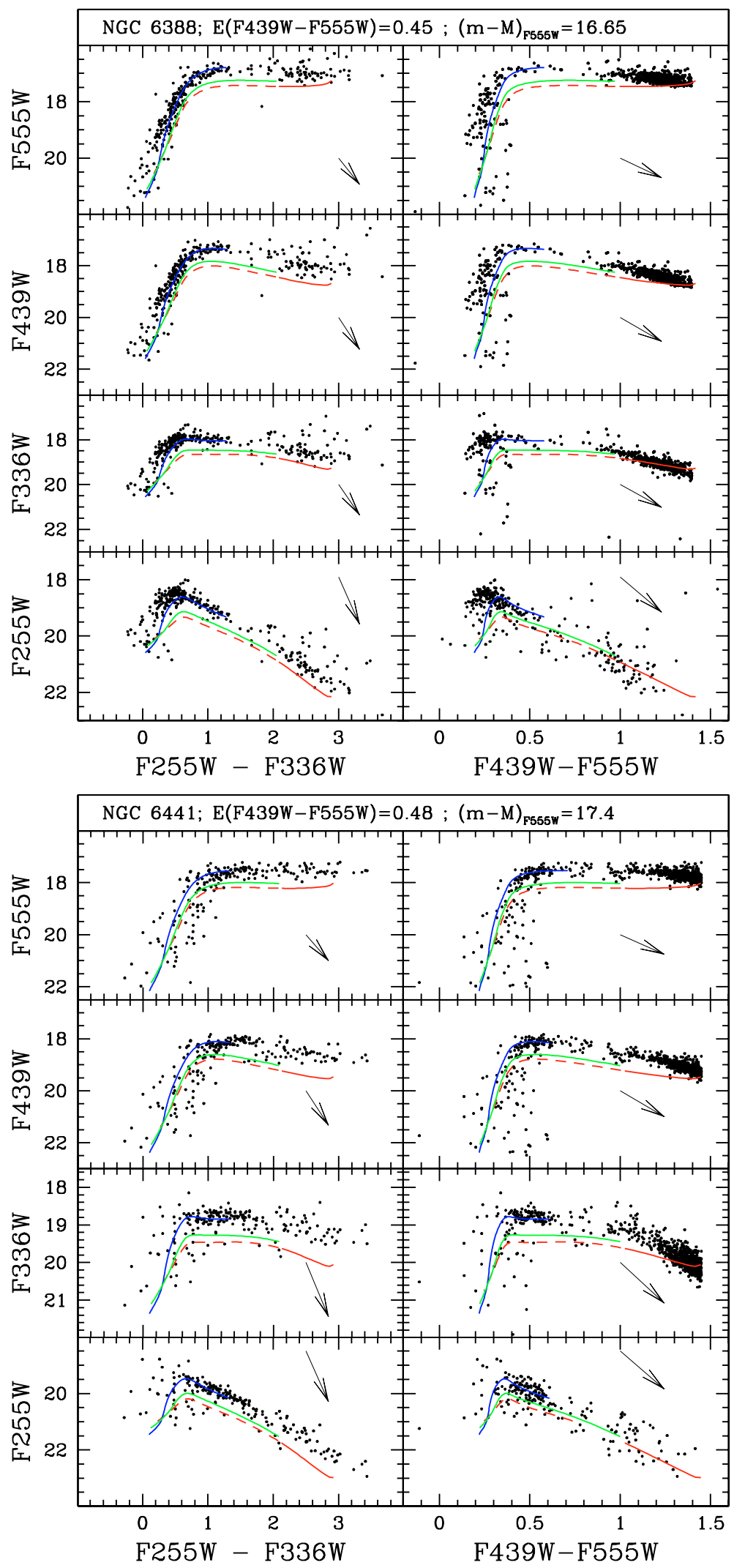

Fig. 6. Top panel: the HBs of NGC 6388 in different photometric planes. The observations are compared with theoretical models for a canonical $Y=0.256$ (red line), for $Y=0.30$ (green line), and for $Y=0.40$ (blue line). The ZAHB models account, in all cases, for a reddening correction dependent on $T_{\text {eff }}$ (see text for details). The shifts applied to the stellar models for the adopted distance modulus and mean reddening are labeled in the figure, and they have been fixed according to our (arbitrary) choice of imposing a best fit between models and the lower envelope of the red HB portion in the $(F 336 W-F 439 W, F 439 W)$ CMD (see Fig. 5). The arrows represent the reddening vector in the different photometric planes. Bottom panel: as in the top panel, but for NGC 6441.

a discontinuous increase of the effective temperature along the $\mathrm{HB}$ at the transition between unmixed (EHF) and mixed (LHF) models, thus producing a gap at the hot end of the HB as indeed observed by Bedin et al. (2000) in NGC 2808, and which could be tentatively related to the possible gap at $F 555 W \approx 19.8$ in the HB of NGC 6388.
From an observational point of view, further support to this scenario was provided by the accurate spectroscopical measurements made by Moehler et al. $(2002,2004)$ for the blue-hook stars belonging to NGC 2808 and Omega Cen. The most important finding was the evidence that blue-hook stars are both hotter and more helium-rich than predicted by canonical models 
of EHB stars. In recent times, the results of Brown et al. (2001) have been fully confirmed on the basis of full self-consistent evolutionary computations performed by Cassisi et al. (2003).

The changes in the surface chemical composition in the LHF structures are really crucial for explaining the fact that the bluehook stars appear fainter than the "normal" hot HB stars. Indeed, LHF stars, as the EHFs, do have smaller He cores at the HeF with respect to canonical models, but the expected smaller core mass implies a reduction in the bolometric luminosity of only $\approx 0.1 \mathrm{mag}$. However, it has been nicely shown by Brown et al. (2001) that LHFs have significantly different spectra with respect to the other stars belonging to the same clusters, but which have not experienced the HeF mixing process. In a normal stellar atmosphere composed mostly of $\mathrm{H}$, the opacity for wavelengths shortward of about $910 \AA$ redistributes the flux in the extreme ultraviolet to longer wavelengths. If $\mathrm{He}$ is enhanced in the stellar atmosphere as in LHF stars, the H opacity source is strongly reduced and so much more flux is emitted in the extreme UV, decreasing the outgoing flux at longer wavelengths. As a consequence, LHF stars should appear fainter in the far and intermediate UV bands (as $F 255 W$ ) compared to EHF and normal HB stars.

It is worth noting that, depending on the properties of the HeF-induced mixing and on the efficiency of the following dredge up, one can expect non-negligible differences in the surface abundances in the sense that some LHF stars might appear more enriched in helium and carbon than others. This occurrence could result in a sizeable spread both in brightness and colors among the LHF stars (as appears to happen in the case of the blue hook candidates in the target clusters).

The empirical data we present for NGC 6388 and NGC 6441 nicely confirm these predictions, and reproduce similar findings in NGC 2808 (Bedin et al. 2000; Brown et al. 2001; Castellani et al. 2006) and Omega Cen (D'Cruz et al. 2000; see also Momany et al. 2004). What is interesting to note in the case of NGC 6388 (for NGC 6441, the number of stars does not allow a firm conclusion) is that the brightness extension of the blue-hook sequence seems to be larger than in the other quoted clusters. It is not possible to clarify, with the present data, whether this occurrence is a signature of a real intrinsic difference between the blue hook stars belonging to the different clusters, or if it is due to the different photometric bands adopted in the various works (see the discussion in Brown et al. 2001), or to photometric errors/contamination by foreground stars, or more simply to higher differential reddening in NGC 6388. In any case, it is evident that an accurate spectroscopic investigation of the blue-hook stars in - at least - NGC 6388, as already done for NGC 2808 and Omega Cen (Moehler et al. 2002), is mandatory. Moehler \& Sweigart (2006a,b) have tried to spectroscopically measure at least the temperature and gravity of the hottest stars in NGC 6388, but they had to conclude that crowding might be a severe obstacle for ground-based spectroscopy. Since HB stars are rare in the cluster outskirts, and mixed with bulge objects, a selection of cluster stars (possibly based on proper motions on wide-field images as in Anderson et al. 2006) would be of great help in this kind of investigation.

\section{The tilted HBs}

Another intriguing property of the HBs of NGC 6441 and NGC 6388 is the occurrence of the tilt for which we still lack a convincing explanation. In the following, we wish to briefly summarize the state-of-art of the investigations on this interesting feature, and provide further evidence which could help in interpreting it.

On a purely empirical ground, Raimondo et al. (2002) have shown that a tilted red $\mathrm{HB}$ is a common occurrence among all metal-rich GCs. This feature appears quite more evident in NGC 6388 and NGC 6441 since these clusters are the only ones showing a blue extension of their HB. On the other hand, Catelan et al. (2006) seem to find that the red HB is more strongly sloped in NGC 6388 than in 47 Tuc over the same color range (their Fig. 3). The most simple explanation for the occurrence of such a feature is to assume that two stellar populations, characterized by distinct metal abundances, exist in these clusters. According to this scenario the blue HB stellar sample would be associated with the metal-poor population, while the red clump would be produced by the metal-rich component.

However, this possibility has been completely ruled out by both the analysis performed by Raimondo et al. (2002), who showed that in both clusters there is not a corresponding sizeable number of RGB stars blueward of their RGB (see also Catelan et al. 2006), and by the spectroscopic measurements of individual metal abundances of RGB stars performed by Carretta (private communication) and Gratton et al. (2006). In NGC 6388, Carretta has found an average metallicity of $[\mathrm{Fe} / \mathrm{H}]=-0.44$, with no evidence of a metallicity spread among the 7 RGB stars for which high-resolution UVES@VLT spectra have been obtained. This result has also been recently confirmed by Wallerstein et al. (2007). Again, from high-resolution UVES@VLT spectra for 5 NGC 6441 red giants, Gratton et al. (2006) found an average metallicity $[\mathrm{Fe} / \mathrm{H}]=-0.39$, with no indication of a metallicity spread larger than their measurement errors. On the other hand, it must be mentioned that Clementini et al. (2005) have found that out of 11 RR Lyrae in NGC 6441, 9 objects have a remarkably similar metal content $[\mathrm{Fe} / \mathrm{H}]=$ -0.57 , whereas 2 objects have a metallicity $[\mathrm{Fe} / \mathrm{H}] \approx-1.3$, though, in this case, the membership of the RR Lyrae is not certain. In view of the results by Gratton and collaborators, a more accurate analysis of the RR Lyrae membership is necessary in order to obtain a firmer result. In any case, also a metallicity as low as $[\mathrm{Fe} / \mathrm{H}]=-1.3$ could not explain the presence of an EHB in NGC 6441.

Piotto et al. (1997) suggested the possibility that in NGC 6441 and NGC 6388 there are two populations with different ages: the oldest population should be responsible for the blue HB, while the younger one creates the red HB. This possibility would indeed explain the bimodal $\mathrm{HB}$, but it would not be able to account for the tilted HB.

Moreover, quite recently Catelan et al. (2006) provided an accurate CMD for NGC 6388 reaching the cluster mainsequence turn off. On the basis of their photometry, they conclude that the age of this cluster is quite similar (within $1 \mathrm{Gyr}$ ) to that of the prototype metal-rich cluster 47 Tuc, with no clear evidence for the existence of an age spread in NGC 6388.

An attempt to simultaneously interpret the occurrence of the blue tail and of the tilt was made by Sweigart \& Catelan (1998) by suggesting three different non-canonical scenarios: i) a very high initial He content; ii) a spread in the He core mass as due to stellar rotation; iii) deep mixing along the RGB. Each one of these mechanisms can - in principle - explain the presence of both the blue tail and the tilt. However, as shown by Raimondo et al. (2002), Moehler \& Sweigart (2006b) and Catelan et al. (2006), these non-canonical scenario seem to be ruled out by some clear empirical evidence. The primordial high He content is excluded by (i) the luminosity of the bump along the $\mathrm{RGB}$, and (ii) the ratio between the number of $\mathrm{HB}$ and $\mathrm{RGB}$ 
stars - the so-called R parameter - which are both consistent with a canonical He content (Layden et al. 1999; Cassisi et al. 2003; Salaris et al. 2004).

Fast rotation is an unlike possibility because, in all GCs with blue HBs, no stars hotter than $T_{\text {eff }} \sim 11500 \mathrm{~K}$ have a rotation velocity $v \sin i>10 \mathrm{~km} \mathrm{~s}^{-1}$ (Recio-Blanco et al. 2002). However, Sweigart (2002) has pointed out that blue HB stars could appear as slow rotators as a consequence of an efficient removal of angular momentum from the envelope due to stellar winds induced by radiative levitation. Clearly, more reliable HB stellar models properly accounting for diffusive processes, mass loss and rotation are mandatory in order to fully exploit this issue. In the same time, it would be worthwhile to investigate the properties of hot HB stars via the asteroseismological analysis of those HB stars experiencing non-radial pulsations such as EC 14026 objects and "Betsy" stars (see Kawaler \& Hostler 2005).

Finally, deep mixing along the RGB, often invoked to explain the abundances anomalies observed in cluster RGB stars, has been recently seriously questioned from an observational point of view by the identification of the same anomalies among main- sequence stars (see Gratton et al. 2004, for a discussion), which points towards a primordial origin for the abundance anomalies. Nevertheless, in more recent times, Suda \& Fujimoto (2006) have discussed the possibility that RGB stars in Galactic GCs could be affected by various mechanisms of mixing between the envelope and the interiors. These different modes of mixing would be triggered by close encounters with other stars that RGB stars could experience in the quite dense stellar environment characteristic of many GCs. The simulations made by Suda \& Fujimoto (2006) have suggested that depending on the occurrence of a specific mode of mixing as well as the evolutionary stage of the RGB star experiencing the process, the HB progeny of these stars could populate the hot side of the $\mathrm{HB}$ and also at a luminosity level significantly larger than that predicted by canonical stellar models.

\subsection{A possible solution to the enigma}

An interesting scenario that could help to solve the puzzle of the extended and tilted HBs of NGC 6388 and NGC 6441, has recently emerged from some quite unexpected results that some of us obtained for the Galactic GCs Omega Cen and NGC 2808. As already suspected by Anderson (1997), Bedin et al. (2004) showed that the main sequence (MS) of Omega Cen is splitted into two sequences, and that this double MS is a ubiquitous feature, spread all over the cluster. Even more interestingly, Piotto et al. (2005), by using high-resolution spectra, showed that the bluer MS is more metal-rich than the red one, at odds with any expectation based on stellar evolution theory. The only possibility to account for a bluer and more metal-rich secondary MS (which contains about $25 \%$ of Omega Cen MS stars) is to assume (as already proposed by Bedin et al. 2004; and Norris 2004) that it corresponds to a highly helium-enhanced $(Y=0.38)$ stellar population. Such a population must necessarily reflect a second generation of stars formed from material polluted by intermediate-mass AGB stars, and/or by type II supernovae, and/or massive rotating stars (Caloi \& D'Antona 2005; Maeder $\&$ Meynet 2005; see also discussion in Piotto et al. 2005; and Bekki \& Norris 2006). Since He-rich stars have a lower turnoff mass at a given age, they will populate the blue side of the $\mathrm{HB}$ during the He-burning phase, and they will also be more luminous because of the higher energy produced by the hydrogenburning shell (Lee et al. 2005, and references therein). Both effects would produce a bluer and brighter $\mathrm{HB}$, exactly as observed in NGC 6388 and NGC 6441.

The HB of Omega Cen is too complicated (because of the large spread in metallicity and age) to clearly distinguish the effect of He enhancement in a minor component of its stellar population from the more prominent effects due to age and metal content. Moreover, Omega Cen may well be an extreme example in which ejecta by a first generation of massive stars can be kept inside a Galactic GC, because of its mass. Indeed, Omega Cen is the most massive GC in our Galaxy, and it might well have been much more massive in the past as, because of its orbit, it must have lost a huge amount of mass due to tidal shocks, mainly passing through the bulge (see Altmann et al. 2005, and reference therein).

However, Piotto et al. (2007) have clearly shown that the Omega Cen MS split is not a unique case among GCs: also NGC 2808 shows three distinct main sequences. An anomalous broadening of the MS of NGC 2808 was already indicated by D'Antona et al. (2005). The simplest explanation of this occurrence is that the various sequences are associated to stellar populations characterized by different initial He contents (D'Antona et al. 2005; Piotto et al. 2007). Interestingly enough, NGC 2808 is the Galactic GC with one of the most complex HB structures: a red HB clump, a very extended blue tail and very few RR Lyrae stars (Sosin et al. 1997; Bedin et al. 2000; Corwin et al. 2004). One has also to note that NGC 2808, is the most massive GC after Omega Cen.

Once again, NGC 6388 and NGC 6441 are between the most massive GCs and, because of their location inside the Galactic bulge, they must have been rather more massive in the past (Ree et al. 2002), in order to have managed to survive till now. Indeed, according to Gnedin \& Ostriker (1997), both clusters have a disruption time slightly shorter than a Hubble time, which means that what we see at the present time might be the remnants of much bigger stellar systems. On the other hand, the existence of populations with an enhanced $\mathrm{He}$ content has been proposed recently Kaviraj et al. (2007) also in the case of extragalactic GCs, suggesting that this phenomenon is typical not only of our galaxy but is in fact more general.

It is therefore rather tempting to associate the anomalously blue and anomalously tilted HBs of NGC 6388 and NGC 6441 to a second generation of stars, strongly He-enriched by pollution from massive and/or intermediate-mass stars of the first star formation burst. A similar suggestion that the presence of a population with He enhancement can explain the anomalous $\mathrm{HB}$ of NGC 6388 and NGC 6441 has been recently made also by Caloi \& D'Antona (2007). Note that this scenario is completely different from that early envisaged by Sweigart \& Catelan (1998), i.e. that the HB morphology of NGC 6388 and NGC 6441 may be due to a population of He-enriched stars. Sweigart and Catelan suggested that all stars in these two clusters are He-rich. Now we suggest that a minority of the stellar population of NGC 6388 and NGC 6441 is He-enriched.

In order to verify the possibility that the peculiar $\mathrm{HB}$ morphology could be explained by accounting for the presence in the target clusters of a He-rich stellar population, we have calculated additional sets of low-mass, He-burning models for suitable choices of the initial He content. More in detail, we computed HB models for a metallicity $Z=0.008$ and inital He contents equal to $Y=0.30$ and 0.40 . The mass of the RGB progenitors $\left(\sim 0.7 M_{\odot}\right)$ has been selected in order to fulfill the condition that their age at the RGB tip be equal to about 13 Gyr. It is worth noticing that these models are fully consistent with the other ones computed by adopting $Y=0.256$, as far as both the 
physical input and numerical assumptions are concerned. The stellar models have been transferred from the theoretical plane to the various observational ones by using the same set of color $-T_{\text {eff }}$ relations adopted for the $Y=0.256$ models. It has been recently shown by Girardi et al. (2007) that the impact of an enhanced $\mathrm{He}$ abundance on color $-T_{\text {eff }}$ relations is completely negligible.

In Fig. 6, we show a comparison between the observed CMDs and the theoretical models computed for the various He contents, adopting the same reddenings and distance moduli as explained in Sect. 4.3. These reddenings and distance moduli have been fixed by imposing that the canonical models overlap the red clump of the HB in the optical bands. As already discussed, the models computed by assuming a canonical He content $(Y=0.256)$ are not able to reproduce the blue HB. On the contrary, one can easily see that the ZAHB models corresponding to $Y=0.40$ are in good agreement with the observed distribution of blue HB stars of NGC 6388 in all of the CMDs (top of Fig. 6). The same kind of comparison, but for the case of NGC 6441, is performed in the bottom panels of Fig. 6. In this case, it is evident that the $Y=0.40 \mathrm{ZAHB}$ is slightly brighter than the observed distribution of blue HB stars. From the comparison between empirical data and the ZAHBs computed for various initial He contents, it appears that it is possible to reproduce the brightness of the blue HB star population by assuming a He content of the order of $Y \approx 0.35$, i.e. slightly lower than in NGC 6388. This fact is consistent with the observational evidence that the blue HB is less extended in NGC 6441 than in NGC 6388.

The presence of two distinct stellar populations characterized by two different initial He contents can help in explaining the brightness difference between the red portion of the $\mathrm{HB}$ and the blue component. However, in order to explain the tilted morphology of the whole HB sequence one should also account for the presence of a spread in the He content at the level of about $\Delta Y \approx 0.05-0.06$ (see also Moehler \& Sweigart 2006b; Caloi \& D'Antona 2007).

In passing, we note that the presence of a moderate spread $(\Delta Y \approx 0.02-0.03)$ in the He abundance around the canonical value $(Y \sim 0.26)$ among the red clump stars, by affecting their intrinsic luminosity, could help explain the peculiar tilted distribution of the red HB clump, too.

In order to provide further support to the hypothesis that the peculiar HB morphology in NGC 6388 and NGC 6441 could be explained as due to the existence in both clusters of stellar populations characterized by different He contents, and eventually to estimate the fraction of stars belonging to the He-enhanced stellar population, we performed an analysis based on the computation of synthetic HBs by adopting stellar model sets for different initial He abundances: $Y=0.256,0.35$ and 0.40 .

The details of the computation of synthetic HBs are fully discussed by Castellani et al. (2003). In our synthetic HB simulation, we assume that the cluster members are a mixture of stars with different initial He abundances ${ }^{5}$. More in detail, our synthetic HB simulations have been performed by adopting the following scheme:

- we first generate a HB stellar generation corresponding to a "canonical" He abundance $Y=0.256$, by adopting a Gaussian distribution with a mean mass $M_{\mathrm{HB}}^{0.256}$ and dispersion equal to $\sigma=0.02 M_{\odot}$;

${ }^{5}$ We note that this approach is quite similar to that adopted by Caloi \& D'Antona (2007) for reproducing the HB morphology of NGC 6441.

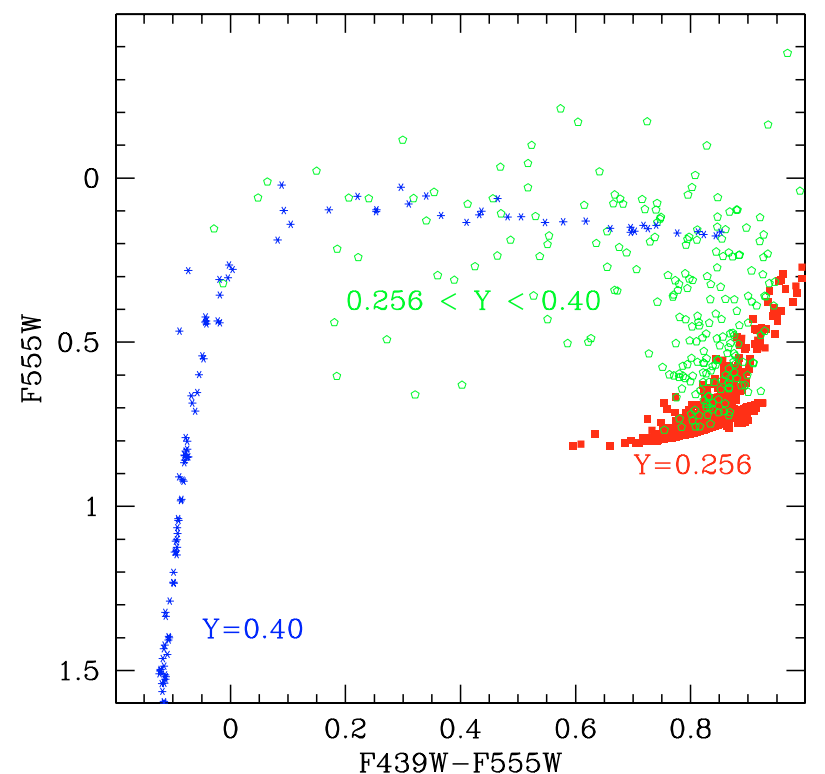

Fig. 7. Synthetic HB simulation for NGC 6388 (see text for more details). The various colors refer to $\mathrm{HB}$ structures with different initial $\mathrm{He}$ content, namely: red color for stars with $Y=0.256$ and blue color for those with He equal to 0.40 , green points correspond to the HB structures whose He abundance is the range $0.256<Y<0.40$.

- a second stellar generation is computed by assuming an initial He content $Y=0.40$, mean mass $M_{\mathrm{HB}}^{0.40}$ and same sigma as above;

- the existence of a stellar population with an initial He abundance between the canonical value and $Y=0.40$ is accounted for by assuming a uniform He content distribution (between $Y=0.256$ and 0.40 ), a Gaussian mass distribution with mean value $M_{\mathrm{HB}}^{-Y-}$ and sigma equal to $0.02 M_{\odot}$. For each He value in the quoted range, the corresponding HB evolutionary tracks used in the synthetic HB simulations have been obtained by linear interpolation between the HB models computed for the previously quoted initial He abundances.

In performing the various simulations we tried to reproduce various observational constraints such as the number of stars along the different portions of the HB (see Table 3) and the number of RR Lyrae stars and their mean pulsational period. It must be clearly stated that, due to the evident large number of free parameters in the simulation, it is not appropriate to attribute too much physical relevance to these simulations ${ }^{6}$, which should be used just to give a general, qualitative picture of the scenario. We think that it is more realistic to consider them as a useful tool to roughly estimate the number of stars in the various stellar populations - for the different initial He contents - that reproduce well the quoted empirical constraints.

In Fig. 7, we show the result of an HB simulation that satisfactorily reproduces the shape ${ }^{7}$, as well as the star counts, of the HB in NGC 6388. This simulation has been performed by adopting: $M_{\mathrm{HB}}^{0.256}=0.65 M_{\odot}, M_{\mathrm{HB}}^{0.40}=0.55 M_{\odot}$ and $M_{\mathrm{HB}}^{-Y-}=0.65 M_{\odot}$. It provides 220 objects in the blue side of the HB, 25 RR Lyrae and 1061 stars in the red side of the HB. These numbers appear

6 As early noticed by Caloi \& D'Antona (2007), the number of RR Lyrae stars as well as their mean pulsational period are largely dependent not only on the choices made for the synthetic simulation but also on the random number extraction.

7 The present simulations do not account for photometric errors as well as for differential reddening. 


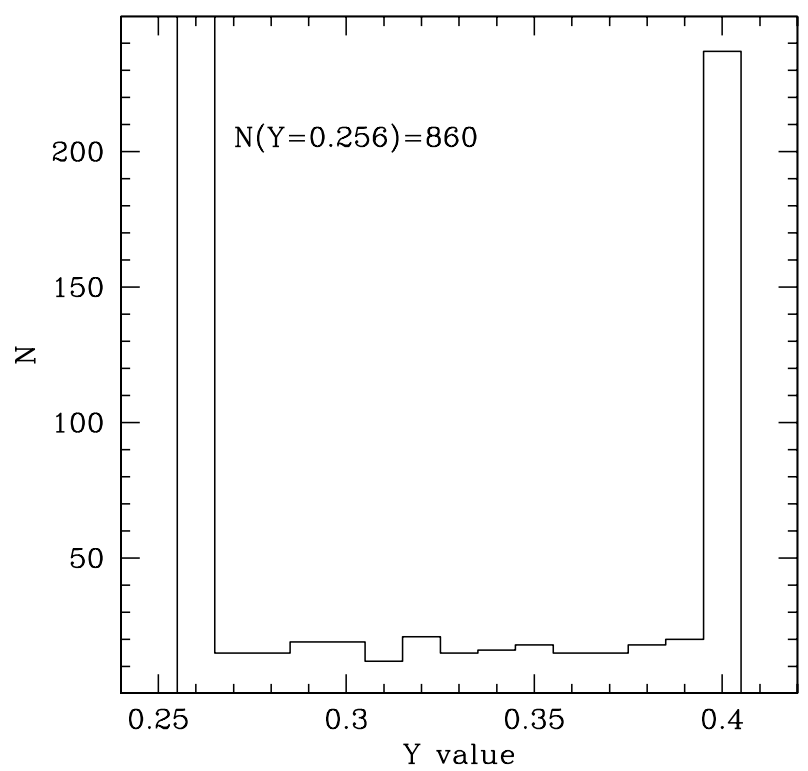

Fig. 8. The number distribution of $\mathrm{HB}$ objects with the initial He content, corresponding to the synthetic HB simulation showed in the previous figure. The total number of objects corresponding to the "canonical" He content $N(Y=0.256)$ is also labeled.

in good agreement with those reported in Table 3 . The distribution of stars with the initial He content is shown in Fig. 8: there are about 860 objects in the peak corresponding to the canonical He content, 230 stars for a He content equal to $Y=0.40$, and 240 objects whose He content is in the range 0.256-0.40. The mean RR Lyrae pulsational period we obtain from this simulation is equal to $P_{\mathrm{RR}} \sim 0.85 \mathrm{~d}$.

The previous analysis has shown that it would be possible to explain the various observational peculiarities of the HBs in NGC 6388 and NGC 6441 by accounting for the presence in both clusters of distinct stellar populations characterized by two different initial He contents: the canonical value $Y \sim 0.26$ and a quite large abundance $Y \sim 0.40$; plus an additional stellar population whose initial He content is - more or less uniformly spread between the two extreme values.

At the beginning of this section, we noted that the clearest, empirical support to the existence in a GC of multiple stellar populations with different He content is the splitting of the MS observed in the clusters Omega Cen and NGC 2808. Therefore, one could argue if the same circumstantial evidence could be observed in the two target clusters. We really think that it is extremely challenging to obtain a similar result for the two target clusters for the following reasons: 1) they are both affected by a sizeable amount of differential reddening; 2) the full interpretation of the $\mathrm{HB}$ morphology would require the presence of some amount of spread in the initial He content between the different stellar populations. Both processes affect the CMD location of MS stars with the global effect of ma it a thorny problem to detect any splitting of the MS locus. In any case, it would be extremely worthwhile to face this difficult task.

\section{Summary and discussion}

We have presented multiband optical and UV CMDs of the Galactic GCs NGC 6388 and NGC 6441. We focused our attention on their anomalous HBs, which show a number of similarities, but also some interesting differences.
The results of the analysis carried out in the previous sections can be summarized as follows:

- The main peculiarities of the HBs are: (i) their extension to very hot temperatures, including (at least for NGC 6388) a number of blue hook candidates, which probably correspond to late helium flasher stars; (ii) the presence of a tilt in the HB.

- The peculiar HBs of NGC 6388 and NGC 6441 cannot be reproduced by any canonical models.

- Reddening and differential reddening contribute to create a sloped HB, but neither of these effects is sufficient to explain the observed tilted HB.

- The presence of a He-rich stellar component - with initial He content in mass $Y$ larger than 0.35 - allows to explain the observational evidence of a blue $\mathrm{HB}$, and the fact that the blue HB in both clusters is brighter than the red HB clump. The presence of a He spread between the "canonical" value and $Y \sim 0.35-0.40$ allows to explain the observed upward slope of the HB in both clusters.

- A comparison with theoretical models computed by adopting various initial He contents suggests that in both NGC 6388 and NGC 6441 the difference in brightness between the red and blue sides of the HB is consistent with the presence in the cluster of a stellar component with a He content as high as $Y \approx 0.40$ in NGC 6388 and $Y \approx 0.35$ in NGC 6441. In addition, in order to explain the whole tilted morphology of the HBs in these clusters, one has to invoke the presence of an additional stellar population characterized by a spread between the two extreme values - of the initial He content. On the basis of a suitable simulation of synthetic HBs for NGC 6388, we estimate that the more He-enhanced stellar population in this cluster should contribute to about $18 \%$ of the total stellar population ${ }^{8}$. Similarly, the stellar population with initial $\mathrm{He}$ abundance in the range $0.26<Y<0.40$ should contribute about $16-17 \%$ of the global population.

- The tilted morphology of the red part of the HBs could also be explained as due to the combined effects of: differential reddening, existence of a (small) He spread among the red clump stars.

- We think that a firmer interpretation of all of the empirical findings presented in the current paper can be achieved only when the reliability of the color $-T_{\text {eff }}$ relation and the bolometric correction scale for metal-rich, cool stars is carefully investigated, as well as when a firmer understanding of the processes regulating the efficiency of mass loss along the RGB is achieved.

In the framework of the scenario proposed in this work, the presence of a He-enriched population implies that in both clusters there must have been at least two episodes of star formation, with the second generation of stars polluted by material ejected from the first formed stars: for the possible culprits, see the scenario described by Ventura et al. $(2001,2002)$ for GCs in general and the discussion for the case of Omega Cen in Piotto et al. (2005), Bekki \& Norris (2006) and Karakas et al. (2006). The main stars responsible for the pollution could be type II supernovae, rotating massive stars, and intermediate-mass AGB stars. Of course, it remains to be understood how the ejected material can remain inside the potential well of the cluster (and it appears not enriched in metals as it should be if the He-enriched matter is provided by type II supernovae). This problem is beyond the

\footnotetext{
8 On the basis of the HB star counts, we estimate that the fraction of He rich stars in NGC 6441 should be lower, i.e. of the order of $10 \%$.
} 


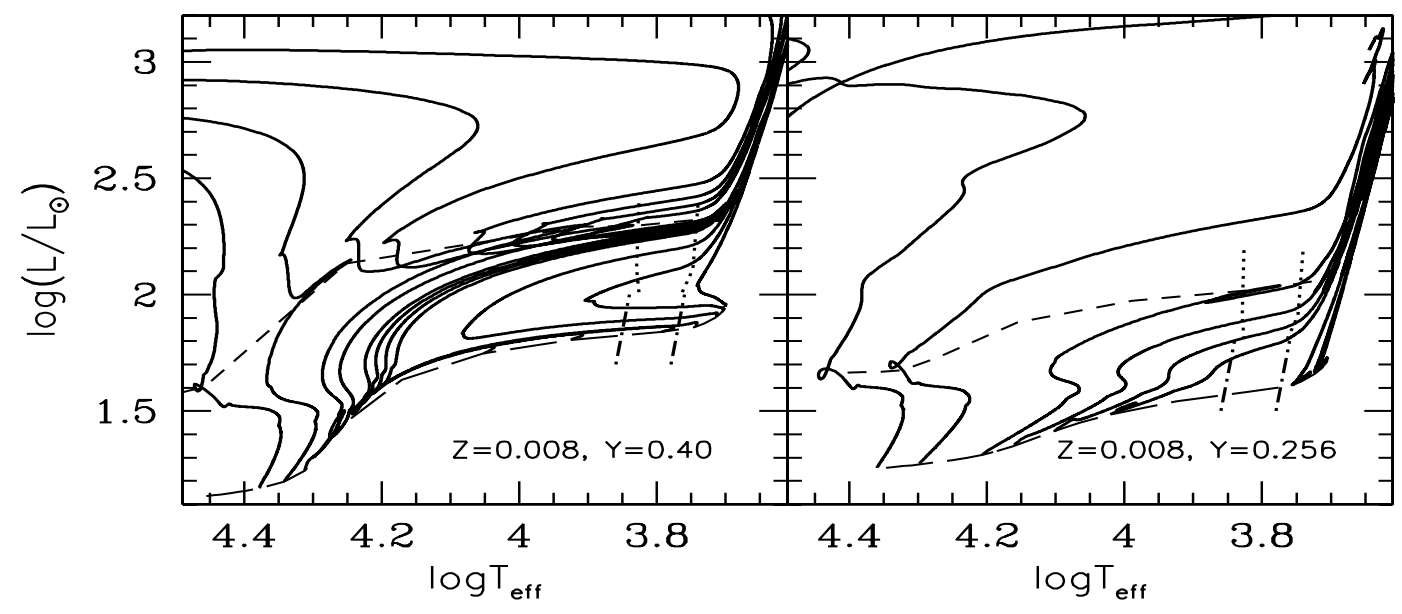

Fig. 9. Left panel: the H-R diagram for selected low-mass, He-burning models for $Z=0.008$ and $Y=0.40$. The stellar masses are (in solar unit): $0.47,0.48,0.49,0.50,0.52,0.53,0.54,0.55,0.60,0.65,0.70$. The ZAHB locus (long dashed line) and the central He-exhaustion one (short dashed line) are shown. The blue and red edges of the RR Lyrae (dot-dashed line) and type II Cepheids instability strip (dotted line) are also plotted. The luminosity of the instability strip boundaries have been - arbitrarily - increased by 0.2 dex in order to account for the larger He content. Right panel: as left panel but for $Y=0.256$. In this case the evolutionary tracks for the 0.47 and $0.48 M_{\odot}$ models are not present. The luminosity levels of the instability strips shown are those reported in literature.

purposes of the present paper. However, it is important to note that, after Omega Cen and NGC 2808, NGC 6388 and NGC 6441 are amongst the most massive clusters of the Galaxy. Because of their position within the bulge, it is conceivable that what we observe at the present time are just two remnants of much bigger systems, which lost part (most?) of their stellar populations via successive tidal shocks, mainly with the Galactic bulge, as expected also from their disruption time, smaller than a Hubble time.

By using synthetic HB models, we have already shown that the presence of multiple stellar populations with different initial He contents could account for many empirical facts related to the peculiar morphology of the HB in both clusters. However, as already noticed, in these simulations there are many free parameters such as the mean HB mass and the mass dispersion for each stellar population, as well as the adopted distribution law for the initial He abundance. This drastically reduces the predictive strenght of this approach. Therefore in order to rely on "first principles", we now wish to briefly comment on the differences in the evolutionary tracks corresponding to HB stellar structures with different initial He contents in order to show how the morphology of these tracks alone is able to provide an explanation for some empirical findings. In Fig. 9, we show the evolutionary tracks in the H-R diagram of selected HB models with two different initial He contents, $Y=0.256$ and $Y=0.40$, i.e. the same values adopted in the previous analysis. The selected stellar masses are the same in both panels, with the only difference that for $Y=0.40$ two additional hot HB models have been plotted. In both panels, we show also the ZAHB locus and the locus corresponding to the central He-exhaustion (CHeEX). The boundaries of the RR Lyrae (Bono et al. 1997, the same adopted for the synthetic HB simulations) and type II Cepheid (Di Criscienzo et al. 2007) instability strips are also plotted.

There is an obvious, expected difference in the brightness of the evolutionary tracks due to the difference in the envelope He content and, in turn, in the shell H-burning efficiency. An interesting feature is that, in the case of the $Y=0.40$ models, the luminosity range between the ZAHB and the CHeEX at the level of the instability strip is about $25 \%$ larger than in the case of the $Y=0.256$ models. This occurrence has the important consequence that, if one assumes the same mass distribution along the HB for a He-rich and for a He-normal stellar population, the He-rich one has a higher probability to produce brighter and, in turn, longer-period variables. An additional interesting feature is the evidence that, due to the peculiar morphology of the He-rich stellar tracks (once again related to the high efficiency of the shell H-burning), many stellar models densely populate a region of the $\mathrm{H}-\mathrm{R}$ diagram, within the instability strip, at luminosities around $\log \left(L / L_{\odot}\right) \approx 2.25$. It is important to notice that this occurs before the end of the core He-burning phase, i.e., when their evolutionary lifetimes are not too rapid, and therefore there is a no negligible probability to observe them. All these morphological properties clearly tend to increase the chance, for a He-rich old stellar population, to produce brighter, long-period, variables.

By comparing the morphology of the evolutionary tracks for the different initial He contents with the predicted boundaries of both the RR Lyrae and type II Cepheids instability strip, it appears clear that a He-rich stellar population has a probability to produce a larger number of type II Cepheids than a normal He stellar population: this might provide a simple explanation for the presence in both NGC 6388 and NGC 6441 of a large population of type II Cepheids.

A firmer understanding of this issue would require additional simulations of synthetic HBs. However, as already noticed, in these simulations the number of free parameters is quite large and this significantly hampers the reliability of this kind of investigation. In a forthcoming paper, we will check if the analysis of the various types of variable stars as well as their pulsational properties and period distribution can help to better constrain the scenario outlined in the previous section.

In Sect. 4.3, we discussed the reasons for which we decided to match the observed CMD and the models at the red end of the HB distribution. In the following, we wish to very briefly comment some implications of using a different choice for matching theoretical predictions to the empirical data, as for instance to force the fit at a point blueward of the red HB clump. When using this approach, one obtains that the red HB clump is 
definitively fainter than canonical models and this occurs also for the hottest HB stars 9 .

None of the evolutionary scenarios outlined in Sect. 6 for explaining the HB morphology of NGC 6388 and NGC 6441 is able to predict a fainter location for the red clump, nor can this occurrence be explained as due to (both absolute and differential) reddening on the basis of the evidence previously discussed. If we accept the hypothesis of multiple stellar populations characterized by different initial He content, a fainter location for the red HB clump would require the presence of a stellar population with an He content lower than the canonical one, a possibility that appears highly unreliable if not impossible.

In addition, Catelan et al. (2006), once forced the red HB clump of NGC 6388 to match the HB of 47 Tuc, have found that the two clusters are approximately coeval. Therefore, we are now facing with two possibilities:

- we could assume that the red HB stars of 47 Tuc are affected by some anomalies as those of NGC 6388 - but this appears at odds with all empirical evidence - and then the result obtained by Catelan et al. (2006) is still valid;

- otherwise we could assume that the HB stars in 47 Tuc are "normal" and those in NGC 6388 are affected by some problem, and then they should appear fainter than those in 47 Tuc. However, if this is the case, the age of NGC 6388 should be larger of about $\sim 5-6$ Gyr with respect that of 47 Tuc. This result appears to us quite unreliable.

On the basis of these considerations, we are confident that the approach adopted in present work, as well as in many others in literature, is reliable.

Acknowledgements. We warmly thanks our anonymous referee for her/his pertinent comments and useful suggestions. This work has been partially supported by INAF and MUR. G.B. gratefully acknowledges support from the Deutsche Forschungsgemeinschaft through grant Mo 602/8 and partial support from the ESO Director General Discretionary Fund.. S.C. warmly acknowledge the Instituto Astrofisico de Canarias (Tenerife) for the hospitality he experienced during his stay at IAC. M.C. acknowledges support by Proyecto FONDECYT Regular No. 1071002. We warmly thank W. B. Landsman and S. Moehler for detailed readings of the manuscript and for useful comments.

\section{References}

Altmann, M., Catelan, M., \& Zoccali, M. 2005, A\&A, 439, L5

Anderson, J. 1997, Ph.D. Thesis, Univ. California, Berkeley

Anderson, J., Bedin, L. R., Piotto, G., Singh Yadav, R., \& Bellini, A. 2006, A\&A, 454, 1029

Bedin, L. R., Piotto, G., Zoccali, M., et al. 2000, A\&A, 363, 159

Bedin, L. R., Piotto, G., Anderson, J., et al. 2004, ApJ, 605, 125

Bekki, K., \& Norris, J. E. 2006, ApJ, 637, L109

Bessel, M. S., Castelli, F., \& Plez, B. 1998, A\&A, 333, 231

Bono, G., Caputo, F., Cassisi, S., Castellani, V., \& Marconi, M. 1997, ApJ, 489, 822

Brown, T. M., Sweigart, A. V., Lanz, T., Landsman, W. B., \& Hubeny, I. 2001, ApJ, 562, 368

Busso, G., Moehler, S., Zoccali, M., Heber, U., \& Yi, S. K. 2005, ApJ, 633, L29

Caloi, V., \& D'Antona, F. 2005, A\&A, 435, 987

Caloi, V., \& D'Antona, F. 2007, A\&A, 463, 949

Carretta, E., Bragaglia, A., Gratton, R. G., et al. 2007, A\&A, 464, 967

Cassisi, S., Schlatt, H., Salaris, M., \& Weiss, A. 2003, ApJ, 582, L43

Castellani, M., \& Castellani, V. 1993, ApJ, 407, 649

Castellani, M., Caputo, F., \& Castellani, V. 2003, A\&A, 410, 871

Castellani, V., Iannicola, G., Bono, G., et al. 2006, A\&A, 446, 569

Catelan, M. 2004, in Variable Stars in the Local Group, ed. D. W. Kurtz, \& K. R. Pollard (San Francisco: ASP), ASP Conf. Ser., 310, 113

Catelan, M. 2005, in Resolved Stellar Populations, ASP Conf. Ser. [arXiv:astro-ph/0507464]

9 This occurrence could be not a real problem when considering the possible presence of blue hook stars.
Catelan, M. 2007, to appear in AIP Conf. Proc. [arXiv: astro-ph/0703724] Catelan, M., Borissova, J., Sweigart, A. V., \& Spassova, N. 1998, ApJ, 494, 265 Catelan, M., Stetson, P. B., Pritzl, B. J., et al. 2006, ApJ, 651, L133 Clementini, G., Gratton, R. G., Bragaglia, A., et al. 2005, ApJ, 630, L145 Corwin, T. M., Catelan, M., Borissova, J., \& Smith, H. A. 2004, A\&A, 421, 667 Corwin, T. M., Sumerel, A. N., Pritzl, B. J., et al. 2006, AJ, 132, 1014 D'Antona, F., \& Caloi, V. 2004, ApJ, 611, 871

D’Antona, F., Bellazzini, M., Caloi, V., et al. 2005, ApJ, 631, 868

D’Cruz, N. L., Dorman, B., Rood, R. T., \& O’Connell, R. W. 1996, ApJ, 466, 359

D’Cruz, N. L., O’Connell, R. W., Rood, R. T., et al. 2000, ApJ, 530, 352

Di Criscienzo, M., Caputo, F., Marconi, M., \& Cassisi, S. 2007, A\&A, 471, 893

Dolphin, A. E. 2000, PASP, 112, 1397

Girardi, L., Castelli, F., Bertelli, G., \& Nasi, E. 2007, A\&A, 468, 657

Gnedin, O. Y., Gnedin, \& Ostriker, J. P. 1997, ApJ, 474, 223

Gratton, R. G., Sneden, C., \& Carretta, E. 2004, ARA\&A, 42, 385

Gratton, R. G., Lucatello, S., Bragaglia, A., et al. 2006, A\&A, 455, 271

Grebel, E. K., \& Roberts, W. J. 1995, A\&AS, 109, 293

Grundahl, F., Catelan, M., Landsman, W. B., Stetson, P. B., \& Andersen, M. I. 1999, ApJ, 524, 242

Heitch, F., \& Richtler, T. 1999, A\&A, 347, 455

Harris, W. E. 1996, AJ, 112, 1487

Holtzman, J. A., Burrows, C. J., Casertano, S., et al. 1995, PASP, 107, 1065

Hui-Bon-Hoa, A., LeBlanc, F., \& Hauschildt, P. H. 2000, ApJ, 535, L43

Karakas, A. I., Fenner, Y., Campbell, S. W., \& Lattanzio, J. C. 2006, ApJ, 652, 1240

Kaviraj, S., Sohn, S. T., O’Connell, R. W., et al. 2007, MNRAS, 377, 987

Kawaler, S. D., \& Hostler, S. R. 2005, ApJ, 621, 432

Kurucz, R. L. 2005, Mem. Soc. Astr. It. Suppl., 8, 14

Layden, A. C., Ritter, L. A., Welch, D. L., \& Webb, T. M. A. 1999, AJ, 117, 1313

Lee, Y-.W., Joo, S. J., Han, S-.I., et al. 2005, ApJ, 621L, 57

Maeder, G., \& Meynet, G. 2005, A\&A, 392, 553

Matsunaga, N., \& IRSF/SIRIUS Team 2006, ASP Conf. Ser., in press [arXiv: astro-ph/0610816]

Moehler, S., Sweigart, A. V., Landsman, W. B., Heber, U., \& Catelan, M. 1999, A\&A, 346, 1

Moehler, S., Sweigart, A. V., Landsman, W. B., \& Dreizler, S. 2002, A\&A, 395, 37

Moehler, S., Sweigart, A. V., Landsman, W. B., Hammer, N. J., \& Dreizler, S. 2004, A\&A, 415, 313

Moehler, S., \& Sweigart, A. V. 2006, Bal. Astron., 15, 41

Moehler, S., \& Sweigart 2006, A\&A, 455, 943

Momany, Y., Bedin, R. L., Cassisi, S., et al. 2004, A\&A, 420, 605

Norris, J. E. 2004, ApJ, 612, L25

Origlia, L., \& Leitherer, C. 2000, AJ, 119, 2018

Pietrinferni, A., Cassisi, S., Salaris, M., \& Castelli, F. 2004, ApJ, 612, 168

Pietrinferni, A., Cassisi, S., Salaris, M., \& Castelli, F. 2006, ApJ, 642, 797

Piotto, G., Sosin, C., Djorgovski, S. G., et al. 1997, AUJD, 15, 32

Piotto, G., King, I. R., Djorgovski, S. G., et al. 2002, A\&A, 391, 945

Piotto, G., Villanova, S., Bedin, L. R., et al. 2005, ApJ, 621, 777

Piotto, G., Bedin, L. R., Anderson, J., et al. 2007, ApJ, 661, L53

Pritzl, B. J., Smith, H. A., Catelan, M., \& Sweigart, A. V. 2002, AJ, 124, 949

Raimondo, G., Castellani, V., Cassisi, S., Brocato, E., \& Piotto, G. 2002, ApJ, 569,975

Recio-Blanco, A., Piotto, G., Aparicio, A., \& Renzini, A. 2002, ApJ, 572, L71

Ree, C. H., Yoon, S. J., Rey, S. C., \& Lee, Y. W. 2002, ASP Conf. Ser., 265, 101

Rich, R. M., Sosin, C., Djorgovski, S. G., et al. 1997, ApJ, 484, L25

Salaris, M., Riello, M., Cassisi, S., \& Piotto, G. 2004, A\&A, 420, 911

Savage, B. D., \& Mathis, J. S. 1979, ARA\&A, 17, 73

Silbermann, N. A., Harding, P., Madore, B. F., et al. 1996, ApJ, 470, 1

Sosin, C., Dorman, B., Djorgovski, S. G., et al. 1997, ApJ, 480, L35

Stetson, P. B. 1987, PASP, 99, 191

Steson, P. B. 1994, PASP, 106, 250

Suda, T., \& Fujimoto, M. Y. 2006, ApJ, 643, 897

Sweigart, A. V. 1997, ApJ, 474, L23

Sweigart, A. V., \& Catelan, M. 1998, ApJ, 501, L63

Sweigart, A. V. 2002, in Highlights of Astronomy 12, ed. H. Rickman (San Francisco: ASP), 92

Ventura, P., D’Antona, F., Mazzitelli, I., \& Gratton, R. 2001, ApJ, 500, L65

Ventura, P., D’Antona, F., \& Mazzitelli, I. 2002, A\&A, 393, 215

Wallerstein, G., Kovtyukh, V. V., \& Andrievsky, S. M. 2007, AJ, 133, 1373

Zoccali, M., Cassisi, S., Bono, G., et al. 2000, ApJ, 538, 289 